\title{
Introduction to the focused section on sensing and perception for autonomous and networked robotics
}

\author{
Xiang Chen $^{1} \cdot$ Mathieu Grossard $^{2} \cdot$ Naoyuki Kubota $^{3} \cdot$ Dirk Wollherr $^{4} \cdot$ Simon X. Yang ${ }^{5} \cdot$ Song Zhang $^{6}$
}

Published online: 5 December 2017

(c) Springer Nature Singapore Pte Ltd. 2017

Next generation of industrial revolution will be featured with broad applications of intelligent technologies; among those popular ones are intelligent manufacturing and autonomous products like vehicles and robotic systems. In both cases, autonomous operations are at the center of the stage, in which appropriate sensing and perception play critical roles. Indeed, recent advances in sensing and perception technologies have produced exciting new ideas in facilitating autonomous manufacturing and/or robotic vehicular systems. These technologies will potentially evolve with more and more 'smart functions' and move manufacturing and robotic systems from single structured operation to sensing/perception-based self-governed yet collaborative multisystem operations. This Focused Section is dedicated to new progresses in modeling, design, control, communication, and

Xiang Chen

xchen@uwindsor.ca

Mathieu Grossard

mathieu.grossard@cea.fr

Naoyuki Kubota

naokubota@nifty.com

Dirk Wollherr

dw@tum.de

Simon X. Yang

syang@uoguelph.ca

Song Zhang

szhang15@purdue.edu

1 Department of Electrical and Computer Engineering, University of Windsor, Windsor, Canada

2 CEA, LIST, Interactive Robotics Laboratory, Fontenay-aux-Roses, France

3 Tokyo Metropolitan University, Hachioji, Japan

4 Technische Universität München, Munich, Germany

5 School of Engineering, University of Guelph, Guelph, Canada

6 School of Mechanical Engineering, Purdue University, West Lafayette, USA implementation of sensing and perception systems for autonomous and/or networked robotics, and intends to provide the state-of-the-art update of research fronts.

The Focused Section consists of six research papers covering detection of human motion (Jiang, et al.), vision based pose measurement (Zhang, et al.), ream-time object detection and tracking (Benabderrahmane), 3-D map reconstruction (Turan et al.; Landsiedel and Wollherr), and vision based endoscopic capsule robot (Turan et al.).

In Jiang et al., an alternative method utilizing temperature fields and their gradients from infrared (IR) images is presented to improve the perception ability of the blind/visually impaired people who are generally familiar with stationary objects but less confident in congested environment where human motion is unpredictable. This approach takes the advantages of the fact that the human body is essentially a natural heat source and is applied to locate individual person and determine his/her face orientation and motion states. This alternative temperature field based perception method is potentially applicable in intelligent space, smart city and smart cars.

In Zhang et al., a global image-to-ground homography based calibration method is presented to obtain the mapping between the image and the planar scene lying in the whole camera field of view, through fusing multiple local homography matrices. The proposed method does not require the knowledge of internal parameters of cameras and renders high calibration accuracy with easy implementation. It can potentially provide sensing aid for mobile robot localization with an accuracy close to the performance limit of a monocular camera.

In Benabderrahmane, an improved real time object detection and tracking framework is presented, built on Adaboost classification, where a strong classifier is generated using an iterative combination of weak learners. A heuristics optimization algorithm is developed to accelerate the extraction of relevant features from the image. Considerable improvement has been observed when applying 
the method to develop an artificial vision for an intelligent transportation system in term of both computing efficiency and accuracy.

In Landsiedel and Wollherr, a method to localize a robot in a global coordinate frame is proposed, based on a sparse 2D map containing outlines of building and road network information with no presumed location information which is obtained from a single 3D laser scan around the robot. The approach extends the generic chamfer matching technique from image processing by including visibility analysis in the cost function. The method is evaluated on two large datasets collected in different real-world urban settings and compared with a baseline method from literature and with the standard chamfer matching approach, where it shows considerably improved performance, as well as the feasibility of achieving global localization based on sparse outline data of buildings.

Then it comes with two papers by Turan et al., in which the effective localization and mapping method is specifically investigated for an endoscopic capsule robot in minimally invasive medical diagnostic applications. The simultaneous localization and mapping (SLAM) based on RGB Depth image fusion is developed in the first paper to provide real-time localization of the capsule robot and the reconstruction of a precise 3D map of the inner organ of human bodies. It is seen that this SLAM method is capable of capturing dense, precise, and globally consistent 3D maps of the explored organ. In the second contribution by the same authors, anther novel localization idea is presented for the endoscopic capsule robot inside the GI tract, which is based on the interpretation of optical flow vector of camera frame pairs with deep learning techniques in order to obtain six degree-of-freedom localization information. It is noted that, in this development, only image information retrieved by a camera is assumed, without the need of extra sensors.

These six papers share a common feature in the work presented, that is, the methodologies developed are all applied to process data generated by sensing devices acting on certain kind of physical fields, such as camera and laser scanning, in order to render 2-D or 3-D information of fields. This kind of field sensing and perception development is the key to facilitate the autonomous operation of robotic systems in practices, which is the main theme of this focused section.

We wish to take this opportunity to extend our sincere gratitude to all of the authors and all anonymous reviewers for their valuable time to make this focused section possible. All of these joint efforts are the critical pushing force to ensure the quality of this focused section. Finally, We wish to thank the Editor-in-Chief, Professor Kok-Meng Lee, for his strong leadership, persistent and strong support, and for all the helps he offered during the whole process of preparing this focused section.

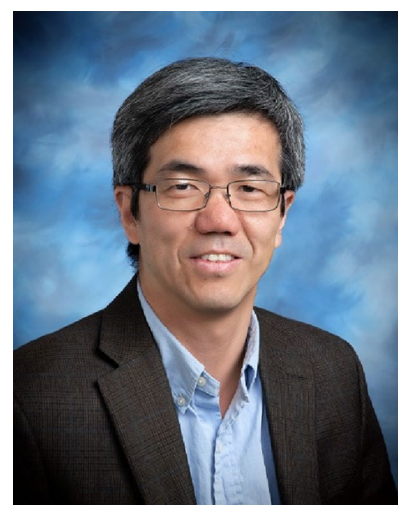

Xiang Chen is currently a Professor in Department of Electrical and Computer Engineering at the University of Windsor, Canada. $\mathrm{He}$ received Ph.D. degree in System and Control from Louisiana State University in 1998. He has been conducting research in robust and nonlinear control theory with application to industrial systems such as automobiles and vision based servo systems. He has made fundamental contribution to control of nonlinear systems with bifurcation and to Gaussain filtering and control. He has also made significant contribution to industrial application in automotive control systems and in vision based sensing systems through extensive collaborative research and development with manufacturing and automotive industries. He is currently a Technical Editor for IEEE/ASME Transaction on Mechatronics and an Associate Editor for SIAM Journal on Control and Optimization. His current research interests include optimization and control of systems with complexities, optimization of field sensor network, and automotive control.

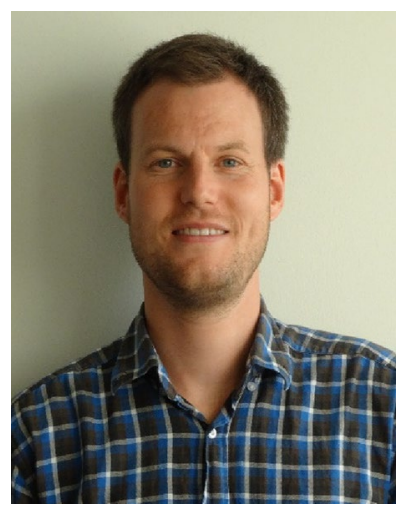

Mathieu Grossard received both the Engineering diploma and the M.Sc. degree in robotics and automation from the Ecole Centrale de Nantes, France, in 2005, and the Ph.D. degree in automatic control (best French PhD award from the "GDR MACS") at Femto-St, France, in 2008. Since 2008, he has been a Researcher and Scientific Project Manager at the Interactive Robotics Laboratory of the CEA LIST in France, where he is involved in research on robotic manipulation and advanced control. In particular, his current research interests include optimal design, modelling and robust control of flexible manipulators, compliant structures, and active-based materials actuators/sensors. He is author or co-author of more than 10 patents in this field, as well as more than 50 International Journals and Conferences. He also has responsibilities in several European-funded research and industrial projects.

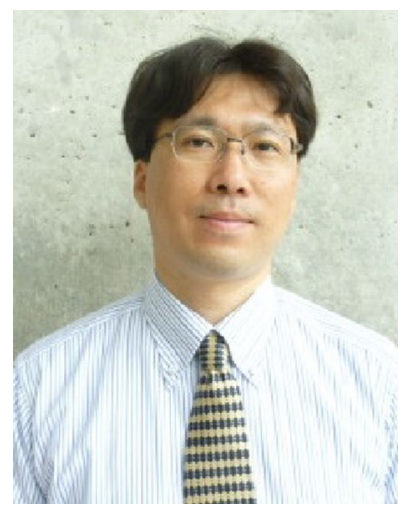

Naoyuki Kubota received the B.Sc. degree from Osaka Kyoiku University, Kashiwara, Japan, in 1992, the M.E. degree from Hokkaido University, Hokkaido, Japan, in 1994, and the D.E. degree from Nagoya University, Nagoya, Japan, in 1997. He joined the Osaka Institute of Technology, Osaka, Japan, in 1997. In 2000, he joined the Department of Human and Artificial Intelligence Systems, Fukui University, as an Associate Professor. He joined the Department of Mechanical Engineering, Tokyo Metropolitan University, in 2004. He is a Professor with the Department of System Design, Tokyo Metropolitan University, 
Tokyo, Japan. His current interests are in the fields of cognitive robotics, robot partners, coevolutionary computation, fuzzy control, spiking neural networks, and informationally structured space.

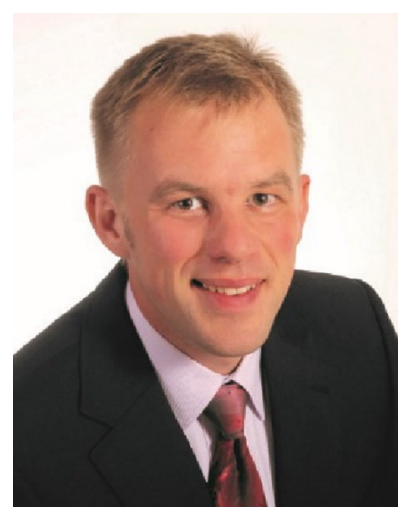

Dirk Wollherr is currently Senior Researcher and Lecturer (since July 2005) and Privatdozent (since 2013) at the Institute of Automatic Control Engineering, Faculty of Electrical Engineering and Information Technology, Technische Universität München, Munich, Germany. He received the diploma engineer degree in Electrical Engineering in 2000, the Doctor of Engineering degree in Electrical Engineering in 2005 and the Habilitation degree in 2013 from the Technische Universität München, Munich, Germany. From 2001 to 2004, he has been Research Assistant at the Control Systems Group, Technische Universität Berlin, Germany. In 2004 he has been granted a Research Fellowship from the Japanese Society for the Promotion of Science (JSPS) at the YoshihikoNakamura-Lab, The University of Tokyo, Japan. His research interests include automatic control, robotics, autonomous mobile robots, humanrobot interaction, and humanoid walking.

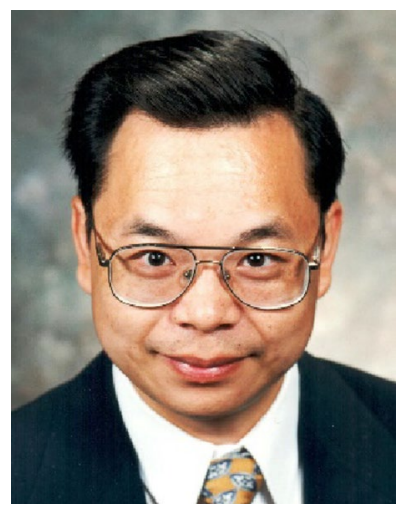

Simon X. Yang received the B.Sc. degree in engineering physics from Beijing University, Beijing, China, in 1987, the first of two M.Sc. degrees in biophysics from the Chinese Academy of Sciences, Beijing, in 1990, the second M.Sc. degree in electrical engineering from the University of Houston, Houston, TX, in 1996, and the Ph.D. degree in electrical and computer engineering from the University of Alberta, Edmonton, AB, Canada, in 1999. Dr. Yang is currently a Professor and the Head of the Advanced Robotics and Intelligent Systems Laboratory at the University of Guelph, Guelph, ON, Canada. His research interests include intelligent systems, robotics, sensors and multi-sensor fusion, wireless sensor networks, control systems, neural networks, fuzzy systems, and computational neuroscience. Prof. Yang has been very active in professional activities. He serves as the Editor-in-Chief of International Journal of Robotics and Automation, Journal of Robotics and Artificial Intelligence, and International Journal of Complex SystemsComputing, Sensing and Control; an Advisory Editorial Board Member of Intelligent Automation and Soft Computing; and an Associate Editor of IEEE Transactions on Cybernetics, and several other journals. He was the General Chair of the 2011 IEEE International Conference on Logistics and Automation, and the Program Chair of the 2015 IEEE International Conference on Information and Automation. Among many of his awards, he was a recipient of the Distinguished Professor Award at the University of Guelph.

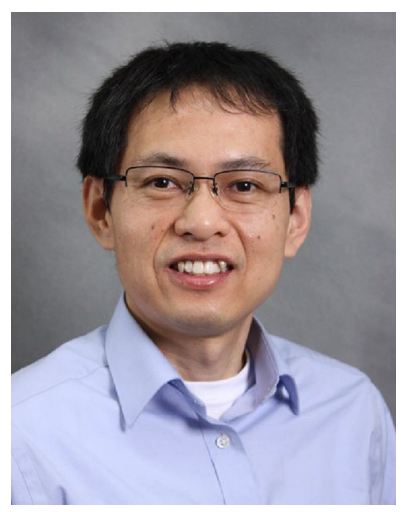

Song Zhang an associate professor of mechanical engineering at Purdue University. He received his Ph.D. degree in mechanical engineering from Stony Brook University in 2005. He was a postdoctoral fellow at Harvard University for 3 years and joined Iowa State University as an Assistant Professor in 2008 and promoted to associate professor in 2014 before moved to Purdue in 2015. He authored on book entitled "High-speed $3 D$ imaging with digital fringe projection techniques", and the editor of another book entitled "Handbook of 3-D machine vision: optical metrology and imaging". He won the AIAA Best Paper Award, Best of SIGGRAPH by the Walt Disney, IEEE ROBIO Best Conference Paper Award, NSF CAREER Award, Stony Brook University's 40 under Forty Alumni Award, College of Engineering Early Career Research Excellence Award from both Purdue University and Iowa State University, and Discovery in Mechanical Engineering Award from Purdue University. He is a fellow of SPIE-the International Society for Optics and Photonics, and a senior member of Optical Society of America (OSA). His current research focuses on developing superfast, super-resolution 3-D imaging technologies and on exploring their applications. 